\title{
A novel compound heterozygous mutation in ABCB4 gene in a pedigree with progressive familial intrahepatic cholestasis 3: a case report
}

\author{
Jie Bai ${ }^{1,2 \#}$, Lu Li ${ }^{1 \#}$, Hui Liu ${ }^{3}$, Shuang Liu $^{2}$, Li Bai ${ }^{2}$, Hanbing Ning ${ }^{4}$, Wenyan Song ${ }^{5}$, Huaibin Zou ${ }^{1}$, \\ Xinxin Wang ${ }^{3}$, Yu Chen ${ }^{1,2}$, Sujun Zheng ${ }^{6}$, Zhongping Duan ${ }^{1,2}$
}

${ }^{1}$ Fourth Department of Liver Disease (Difficult \& Complicated Liver Diseases and Artificial Liver Center), Beijing You An Hospital, Capital Medical University, Beijing, China; ${ }^{2}$ Beijing Municipal Key Laboratory of Liver Failure and Artificial Liver Treatment Research, Beijing, China; ${ }^{3}$ Department of Pathology, Beijing You An Hospital, Capital Medical University, Beijing, China; ${ }^{4}$ Department of Digestive Diseases, the First Affiliated Hospital of Zhengzhou University, Zhengzhou, China; ${ }^{5}$ Department of Radiology, Beijing You An Hospital, Capital Medical University, Beijing, China; ${ }^{6}$ First Department of Liver Disease, Beijing You An Hospital, Capital Medical University, Beijing, China

\#These authors contributed equally to this work.

Correspondence to: Sujun Zheng; Zhongping Duan. Difficult \& Complicated Liver Diseases and Artificial Liver Center, Beijing You An Hospital,

Capital Medical University, No. 8, Xitoutiao, Youwai Street, Fengtai District, Beijing 100069, China.

Email: zhengsujun003@126.com; duan2517@163.com.

\begin{abstract}
Progressive familial intrahepatic cholestasis (PFIC) includes a group of genetic autosomal recessive disorders that predominantly affects young children and results in early-onset progressive liver damage. Variations in $A B C B 4$ have been shown to cause PFIC3. However, the association between $A B C B 4$ genotype and clinical manifestations remains unclear. We investigated the clinical manifestations and genetic features of a Chinese Han pedigree with PFIC3. A 15-year-old boy, with high-serum gamma-glutamyl transferase $(\gamma-$ GT) cholestatic cirrhosis, was diagnosed with PIFC3. After ursodeoxycholic acid (UDCA) treatment, the boy stayed in a relatively stable state with mild itching, and elevated $\gamma$-GT exhibited a remarkable decrease. Genetic testing identified a novel compound heterozygous mutation L842P/V1051A in $A B C B 4$, which was inherited from his mother and father, respectively. Several predictive software suggested that these two mutations are pathogenic. Interestingly, the same compound heterozygous mutation was also found in his two sisters, one of whom had a history of intrahepatic cholestasis of pregnancy (ICP) and the other had asymptomatic gallstones. Therefore, this novel compound heterozygous mutation L842P/V1051A caused a continuum of $A B C B 4$-related diseases including ICP, cholelithiasis and PFIC3 in our pedigree. The inconsistency between genotypes and phenotypes may be influenced by other factors. Genetic testing will be useful for diagnosis and genetic counseling.
\end{abstract}

Keywords: Progressive familial intrahepatic cholestasis (PFIC); compound heterozygous mutation; ABCB4; Liver cirrhosis; case report

Submitted May 06, 2020. Accepted for publication Dec 16, 2020.

doi: 10.21037/atm-20-3747

View this article at: http://dx.doi.org/10.21037/atm-20-3747

\section{Introduction}

Progressive familial intrahepatic cholestasis (PFIC) is an autosomal recessive disorder characterized by early-onset intrahepatic cholestasis (1). It often occurs in infants or children and accounts for $9 \%-13 \%$ of infant cholestasis (2).
PFIC can be divided into 6 types according to different pathogenic genes (3). The pathogenic genes for type 1-6 PFIC are ATP8B1, ABCB11, ABCB4, T7P2, NR1H4 and $M Y O 5 B$, respectively (4-6). For PFIC3, the mutation in $A B C B 4$ gene leads to the deficiency of class III multidrug 
Table 1 Biochemical characteristics of the patient

\begin{tabular}{|c|c|c|c|c|c|c|c|}
\hline Examination item & Reference & 2019.08 & 2019.09 & 2019.10 & 2019.11 & 2020.01 & 2020.03 \\
\hline Direct bilirubin $(\mu \mathrm{mol} / \mathrm{L})$ & $<7$ & 77.5 & 83.5 & 137.8 & 86.7 & 107.5 & 108.2 \\
\hline Alanine aminotransferase (U/L) & $9-50$ & 139.5 & 97 & 108 & 34 & 89 & 96.5 \\
\hline Aspartate aminotransferase (U/L) & $15-40$ & 183.7 & 61 & 65 & 149 & 139 & 162 \\
\hline Alkaline phosphatase (U/L) & $45-125$ & 348 & 330 & 372 & 353 & 372 & 392 \\
\hline Total serum bile acid $(\mu \mathrm{mol} / \mathrm{L})$ & $<10$ & 115.5 & 167.7 & 166.6 & 184.5 & 147 & 113.4 \\
\hline Albumin (g/L) & $36-55$ & 32.9 & 31.8 & 32.2 & 36.2 & 30.4 & 34.9 \\
\hline White blood cell $\left(\times 10^{9} / \mathrm{L}\right)$ & $3.5-9.5$ & 3.41 & 4.28 & 3.82 & 3.69 & 3.96 & 4.2 \\
\hline
\end{tabular}

resistance P-glycoprotein (MDR3), thus affecting the transport of phosphatidylcholine (PC) from the hepatocytes to the bile ducts. The ensuing increase in free bile acids and cholesterol crystals is responsible for the damage of cholangiocyte membranes $(7,8)$. Since PFIC3 does not cause bile acids to stay in liver cells, cholestasis appears late, and progresses slowly. PFIC3 patients usually present with a combination of mild jaundice, pruritus, hepatomegaly and growth retardation. Elevated gamma-glutamyl transferase $(\gamma-G T)$ level is regarded as a serological feature in patients with PFIC3. The characteristic histopathological changes in PFIC3 consist of non-specific portal inflammation, extensive portal fibrosis, and cholestasis with ductular proliferation.

To date, more than 500 mutations in $A B C B 4$ gene have been reported, most of which are missense mutations (9). $A B C B 4$ gene variants are closely associated with several liver diseases such as PFIC3, benign recurrent intrahepatic cholestasis (BRIC), intrahepatic cholestasis of pregnancy (ICP), drug-induced liver injury (DILI), and low phospholipid-associated cholelithiasis (LPAC) $(10,11)$. Homozygous or compound heterozygous mutations in $A B C B 4$ gene used to be considered as characteristic variants in PFIC3 patients, whereas heterozygous mutations in a single allele were reported in patients with LPAC and ICP (11). Nevertheless, the relationship between genotype and phenotype remains to be elucidated, and the molecular mechanisms underlying PFIC3 and other ABCB4-related liver diseases still need to be explored. Herein, we aimed to report a novel compound heterozygous mutation in $A B C B 4$ gene and to investigate the clinical manifestations and genetic features in a Chinese Han pedigree with PFIC3. We present the following article in accordance with the CARE reporting checklist (available at http://dx.doi.org/10.21037/ atm-20-3747).

\section{Case presentation}

The patient (II:3), a 15-year-old boy, was admitted to Beijing You An Hospital in 2019 with the complaint of yellow urine for one year. The boy was in good performance status with a normal BMI at admission. Moderate skin and scleral jaundice were observed. His left breast was slightly enlarged. Physical examination found that the spleen was enlarged with its lower edge $3 \mathrm{~cm}$ above the navel. No signs of lung and heart abnormalities were observed.

Total bilirubin (TB), alanine aminotransferase (ALT), alkaline phosphatase (ALP), total bile acid (TBA) and $\gamma$-GT were elevated (Table 1). PLT count $\left(77 \times 10^{9} / \mathrm{L}\right)$ was decreased. Fasting plasma lipids and immunoglobulins including IgG, $\operatorname{IgA}, \operatorname{IgM}$, and $\operatorname{IgE}$ were normal. Blood coagulation function, autoantibodies profile, anti-neutrophil cytoplasmic antibody (ANCA), and alpha fetal protein (AFP) were also normal.

Contrast-enhanced computed tomography (CT) reported liver cirrhosis, splenomegaly, collateral pathway, and stenosis of portal vein (Figure 1). Gastroscopy revealed severe esophagogastric varices (Figure 2). Histopathology examination showed biliary cirrhosis with ductopenia, ductular reaction, and cholestasis (Figure 3). The handling 

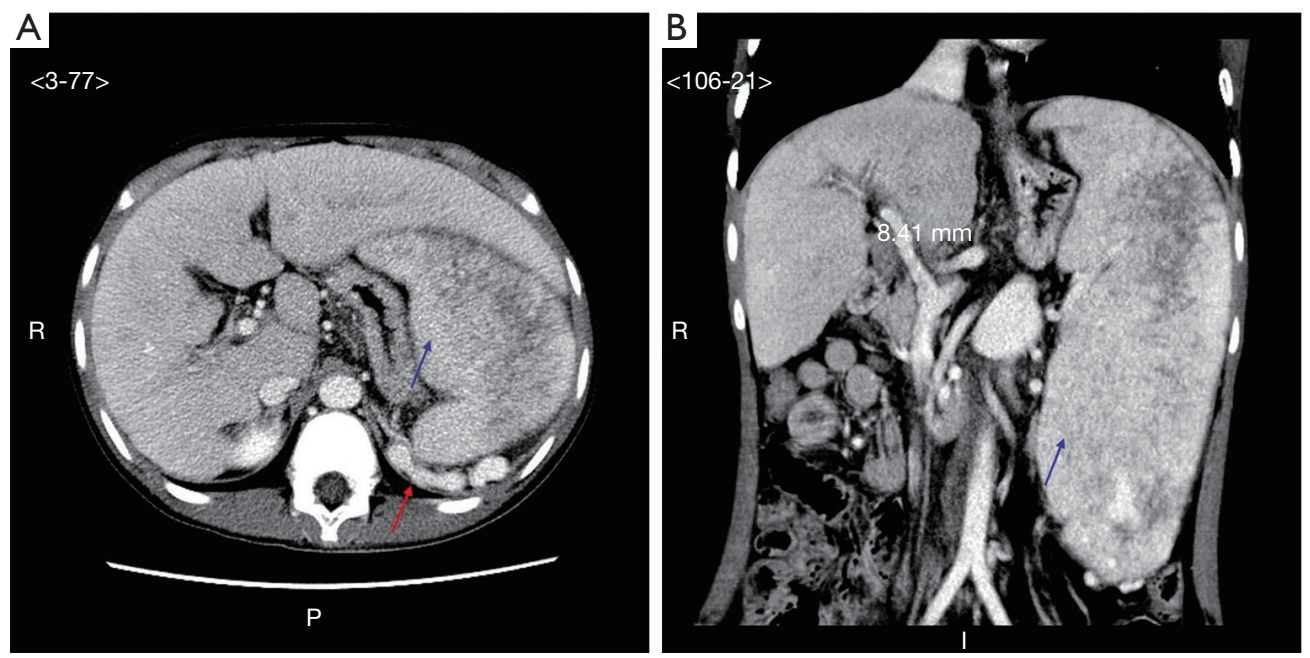

Figure 1 The imaging examination revealed liver cirrhosis, splenomegaly, collateral access and portal vein stenosis. (A) Contrastenhanced CT shows liver cirrhosis, splenomegaly (blue arrow), and collateral pathway (red arrow); (B) the reconstructed MIP image shows splenomegaly (blue arrow) and the portal vein is about $8 \mathrm{~mm}$ in diameter.
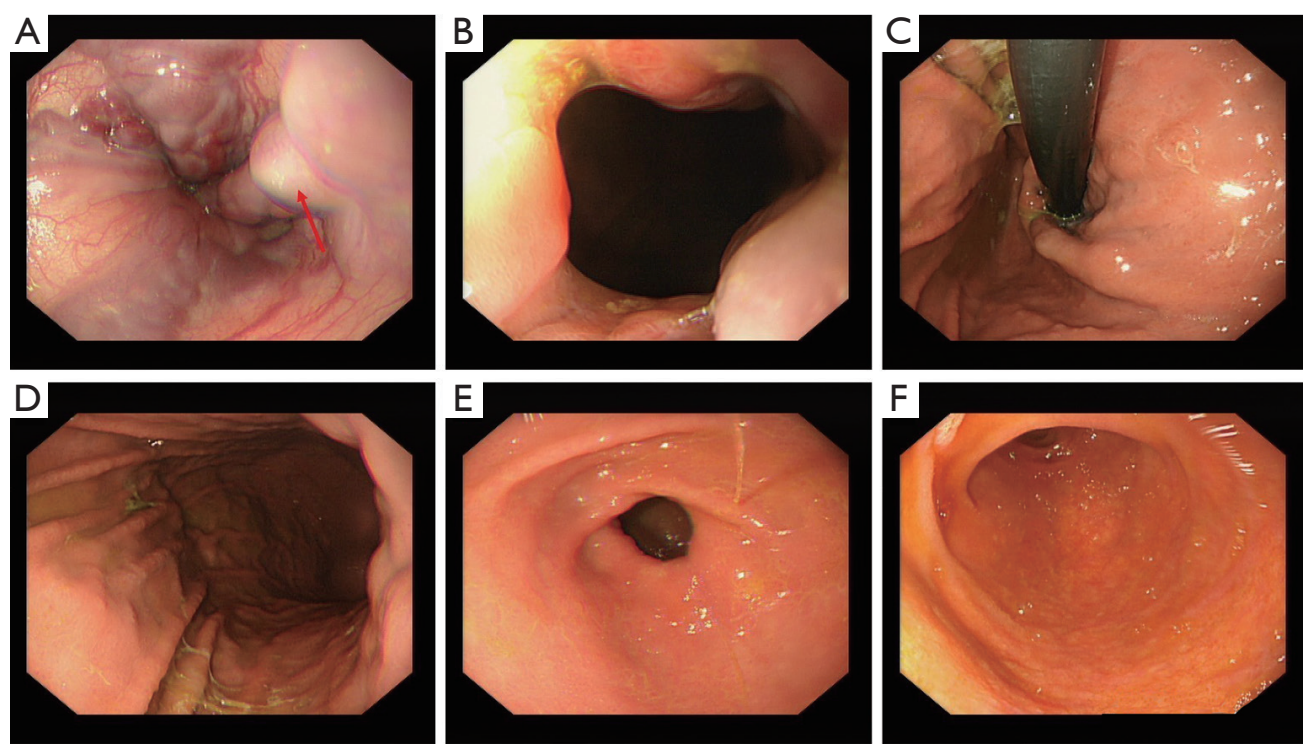

Figure 2 The gastroscopy reveals severe esophagogastric varices. (A) Esophagus; (B) cardia; (C) fundus of stomach; (D) body of stomach; (E) antrum of stomach; (F) descending part of duodenum. The esophagogastric varices are indicated by red arrow.

and staining of liver specimens are performed according to the description by Suriawinata et al. (12).

The patient was diagnosed with PFIC3 in terms of jaundice, cholestasis, persistent pruritus, and elevated $\gamma$-GT. He has been undergoing therapy with ursodeoxycholic acid (UDCA) and cholestyramine. After therapy, the boy stayed in a relatively stable state with mild itching, and elevated $\gamma$-GT exhibited a remarkable decrease (Table 1).
We also investigated the family members of the patient. His two sisters were in their 20s. An elder sister (II:3) was diagnosed as ICP at the age of 17 (Figure 4). Pruritus and increased levels of ALT (104 U/L), AST (82 U/L), TB $(25.8 \mu \mathrm{mol} / \mathrm{L})$, and TBA $(49.7 \mu \mathrm{mol} / \mathrm{L})$ were reported. The relieved symptom and improved liver function were noticed after the treatment with UDCA for 20 days. All abnormalities had completely resolved after discharge. 

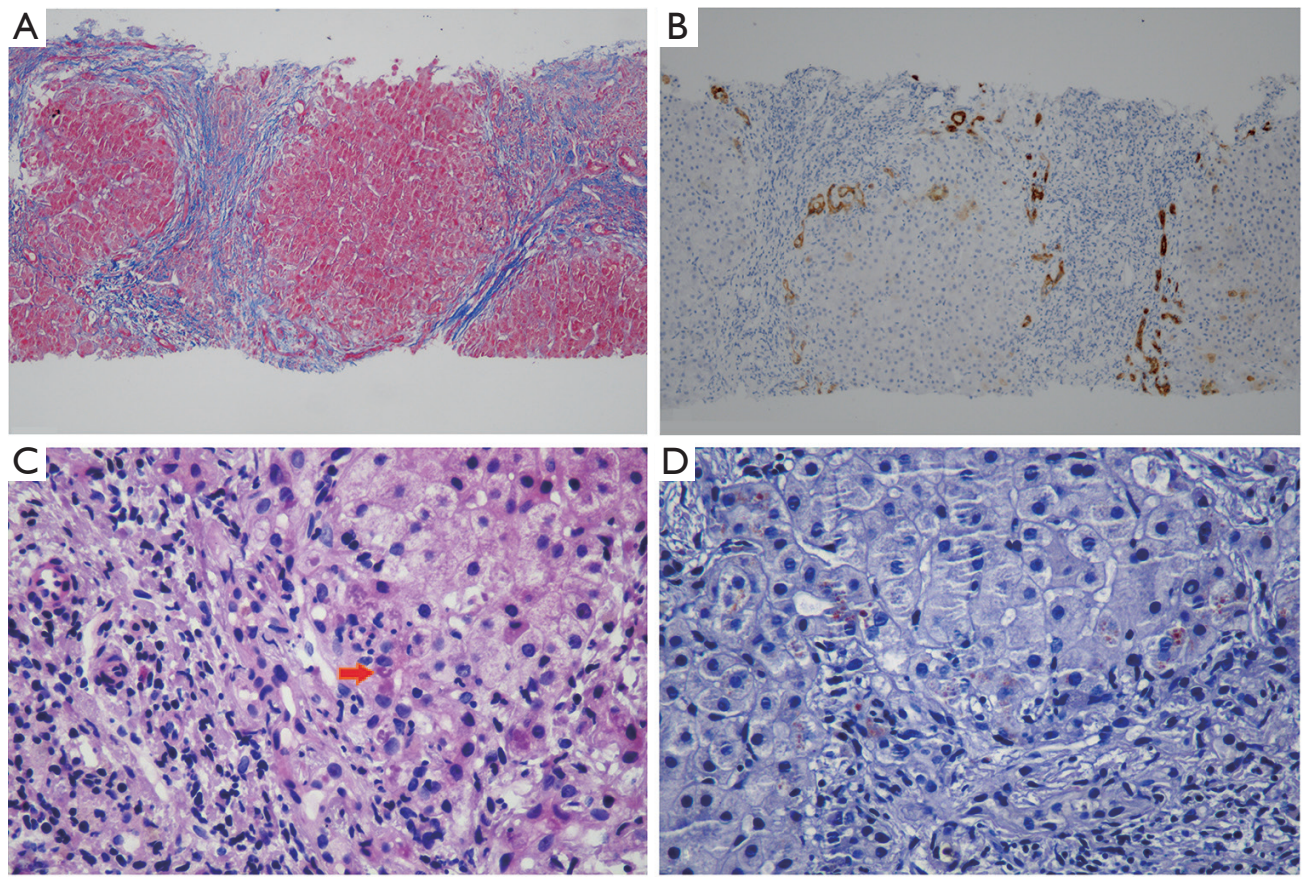

Figure 3 The liver histopathology reveals biliary cirrhosis. (A) Cirrhosis demonstrated by Masson trichome staining (Masson trichome 100x); (B) Ductopenia and ductular reaction (Immunostaining for CK-7, 100x); (C) Cholate stasis and formation of Mallory-Denk bodies (red arrow) (H\&E 400x); (D) Copper accumulation (Rhodanine 200x) in periseptal liver cells.

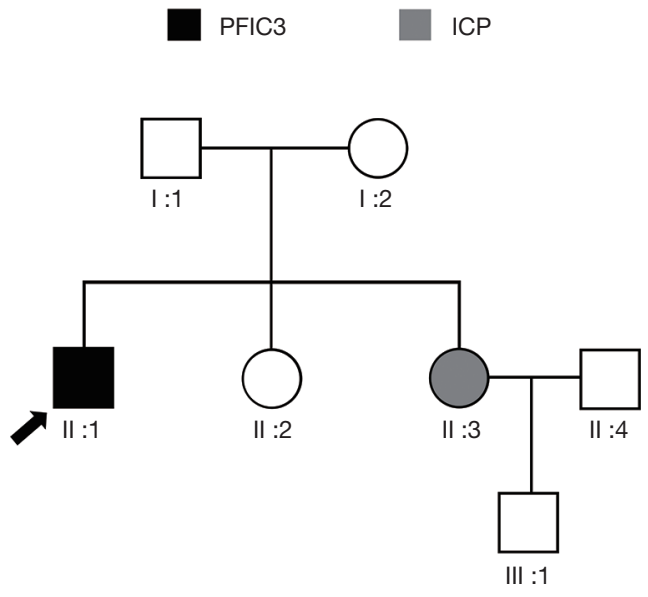

Figure 4 Pedigree chart of the patient's family. The arrow indicates the patient. PFIC3, progressive familial intrahepatic cholestasis; ICP, intrahepatic cholestasis of pregnancy.

Another sister (II:2) had asymptomatic gallstones. His mother suffered from gallstones and underwent cholecystectomy. The history of ICP had been denied. Other family members had no PFIC-related symptoms and no abnormalities in physical examination.

\section{Genetic testing results and bioinformatics analyses}

To explore the genetic factor for PFIC3, panel sequencing of major genes involved in liver metabolism was performed in the patient (II:1). A total of 289 genes were detected, including the common PFIC-related genes ATP $8 B 1$, $A B C B 11$ and $A B C B 4$. As a result, a novel compound heterozygous mutation c.T2525C (p.L842P)/c.T3152C (p.V1051A) in $A B C B 4$ (isoform A) was identified. These two mutations were further confirmed by Sanger sequencing (Figure 5). Seven pieces of software were utilized to predict the pathogenicity of these two gene mutations. As shown in Table 2, all software except for InterVar showed that the mutations L842P and V1051A were pathogenic or damaging. The PhastCons scores of these two mutations were both 1 , and the corresponding PhyloP values were 4.82 and 3.425 , respectively, suggesting the high conservation of these two amino acids.

The known molecular structure of $A B C B 4$ was obtained from Protein Data Bank (Primary Citation of Related Structures: 6S7P) (13), as shown in Figure 6A. The mutated $A B C B 4$ structures were constructed by SWISS-MODEL 


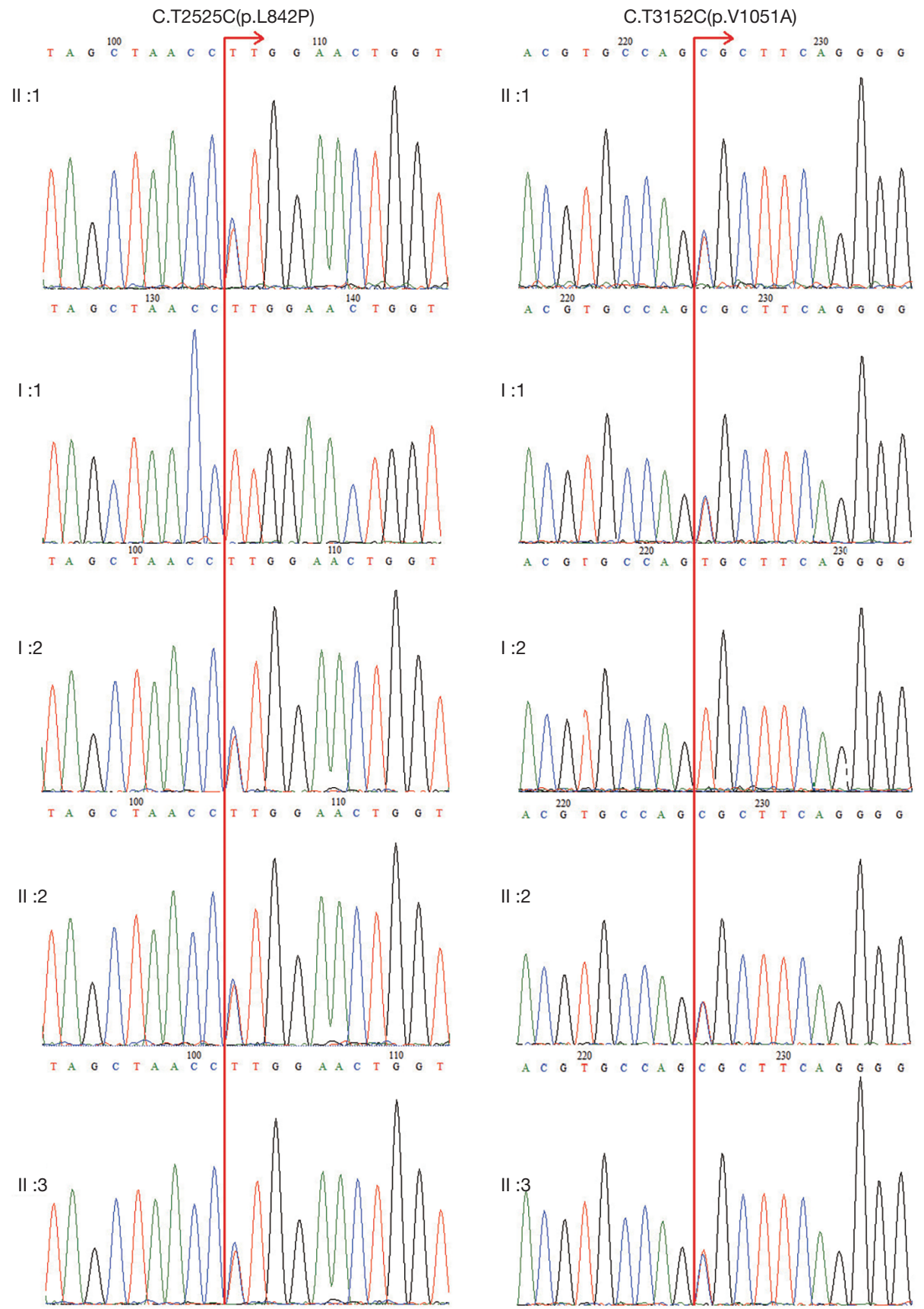

Figure 5 Sanger sequencing reveals two heterozygous mutations in $A B C B 4$ gene, c.T2525C(p.L842P) and c.T3152C(p.V1051A).

(https://swissmodel.expasy.org/interactive). And molecular graphics were analyzed by Swiss-Pdb Viewer 4.1.0 software (Figure 6B). In the predicted mutation structures, the secondary structure of this protein was not affected by the substitution of these two amino acids.

Moreover, we tested the mutations in $A B C B 4$ gene for other family members. The same compound heterozygous mutation was also found in his two sisters (Figure 5). In addition, his mother (I:2) carried the variant L842P, whereas his father (I:1) was a carrier of the other variant V1051A (Figure 5). No other $A B C B 4$ gene variants were observed in this pedigree. 
Table 2 Bioinformatics analysis of the $A B C B 4$ mutations

\begin{tabular}{|c|c|c|c|c|c|c|c|c|c|c|c|c|}
\hline Mutation & \multicolumn{2}{|c|}{ PolyPhen2 } & \multicolumn{2}{|r|}{ SIFT } & \multicolumn{2}{|c|}{ PROVEAN } & \multicolumn{2}{|c|}{ MutationTaster } & \multicolumn{2}{|c|}{ FATHMM } & $\frac{\text { InterVar }}{\text { Prediction }}$ & $\frac{\text { MutPred2 }}{\text { Score }}$ \\
\hline $\begin{array}{l}\text { c.T2525C } \\
\text { (p.L842P) }\end{array}$ & 0.998 & $\begin{array}{l}\text { Probably } \\
\text { damaging }\end{array}$ & 0.001 & Deleterious & -5.86 & Deleterious & 1 & $\begin{array}{l}\text { Disease } \\
\text { causing }\end{array}$ & -2.62 & Damaging & $\begin{array}{l}\text { Uncertain } \\
\text { significance }\end{array}$ & 0.879 \\
\hline $\begin{array}{l}\text { c.T3152C } \\
\text { (p.V1051A) }\end{array}$ & 0.818 & $\begin{array}{l}\text { Possibly } \\
\text { damaging }\end{array}$ & 0 & Deleterious & -3.81 & Deleterious & 1 & $\begin{array}{l}\text { Disease } \\
\text { causing }\end{array}$ & -2.78 & Damaging & $\begin{array}{l}\text { Uncertain } \\
\text { significance }\end{array}$ & 0.530 \\
\hline
\end{tabular}
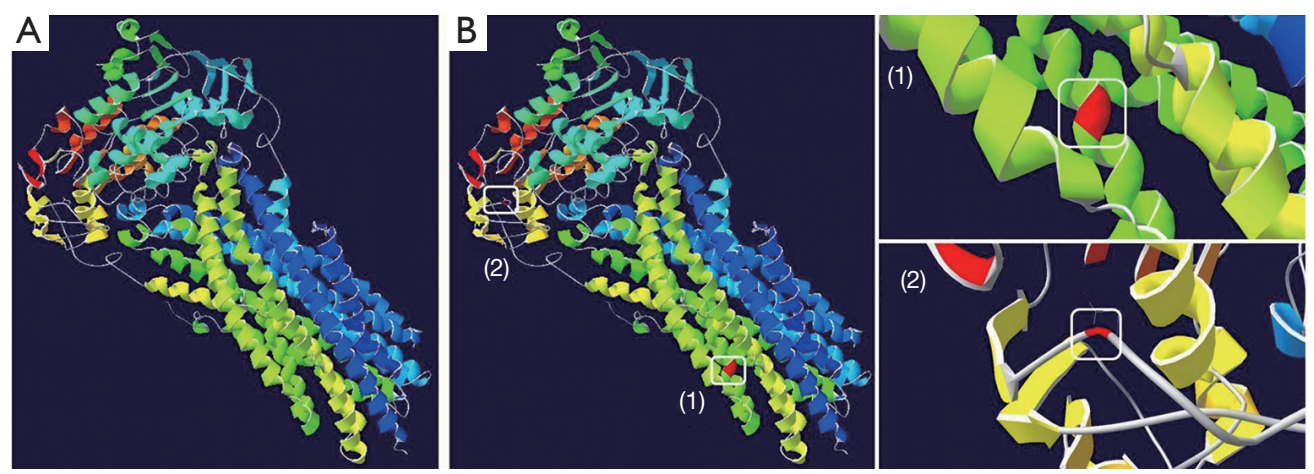

Figure 6 3D structure of $A B C B 4$. (A) Wild-type; (B) mutant type, (1) for c.T2525C(p.L842P) and (2) for c.T3152C(p.V1051A). The structure of $A B C B 4$ was obtained from Protein Data Bank (Primary Citation of Related Structures: 6S7P). And the mutant structures of ABCB4 were constructed by SWISS-MODEL (https://swissmodel.expasy.org/interactive). Molecular graphics were analyzed by Swiss-Pdb Viewer 4.1.0 software.

\section{Ethical statement}

All procedures performed in studies involving human participants were in accordance with the ethical standards of the Institute Ethical Committee of Beijing You An Hospital, Capital Medical University, Beijing, China and with the Helsinki Declaration (as revised in 2013). Written informed consent was obtained from the patients for publication of this manuscript and any accompanying images.

\section{Discussion}

PFIC3 is an autosomal recessive disorder characterized by early-onset cholestasis and progressive liver damage caused by severe mutations in the $A B C B 4$ gene $(7,8)$. In the present study, we describe a Chinese Han pedigree suffering from PFIC3. To our knowledge, this is the first time to report a novel compound heterozygous mutation L842P/V1051A in $A B C B 4$ gene in a 15 -year-old PFIC3 patient. In addition, family members who share the same $A B C B 4$ genotype with the patient exhibit completely different phenotypes, namely, ICP or asymptomatic gallstones.
Human $A B C B 4$ gene is located on chromosome $7 \mathrm{q} 21.1$ and encodes the hepatobiliary PC floppase MDR3. In patients with MDR3 deficiency, the accumulation of nonmicellar, free bile acids may lead to injured cholangiocytes. MDR3 deficiency in PFIC3 can be ascribed to the mutation of two alleles (biallelic variants) in the $A B C B 4$ gene, of which missense variants are the most popular. Furthermore, frameshift variants and splicing mutations in $A B C B 4$ have also been reported $(14,15)$. Disease severity depends on the type of mutations and the remaining function of the transporter protein (16). Dröge et al. have shown that four common ABCB4 SNPs c.175C>T (p.L59L), c.504C>T (p.N168N), c.711A>T (p.I237I) and c.1954A>G (p.R652G) may contribute to cholestasis (17). For those patients without disease-causing mutation, $19 \%$ of them display the combinations of the common synonymous variant c. $711 \mathrm{~A}>\mathrm{T}$ with two other common polymorphisms c. $175 \mathrm{C}>\mathrm{T}$ and c.504C $>\mathrm{T}$ (17). In our pedigree, the patient exhibited typical manifestations of PFIC3 (jaundice, cholestasis, and pruritus) accompanied by elevated serum $\gamma$-GT levels. Panel sequencing of 289 genes involved in liver metabolism identified a novel compound 
heterozygous mutation L842P/V1051A in $A B C B 4$ gene in the patient, which was inherited from his mother and father, respectively. $A B C B 4$ variants detected by NGS were confirmed by Sanger sequencing. Most predictive software except for InterVar suggested that these two mutations are pathogenic. InterVar is a semi-automatic bioinformatics tool. It is helpful for clinical interpretation of genetic variants by algorithms different from other software. This may account for, at least in part, the difference in predictive results. In addition, PhastCons and PhyloP indicated the high conservation of these two amino acids, suggesting that these two mutations have a great impact on the function of proteins. These results show that these two mutations are responsible for $\mathrm{PFIC} 3$ in this patient.

$A B C B 4$ consists of two ATP-binding cassettes, called the nucleotide binding domain (NBD), and six transmembrane-spanning segments, known as transmembrane domain (TMD). The TMDs form the translocation pathway across biofilms. Two TMDs form the required functional unit together with energy, which is provided by ATP binding and/or hydrolysis within the NBDs, thus allowing substrate transport against a concentration gradient (10). The mutation L842P is located in TMD, while V1051A is located in NBD. At present, the pathogenesis of these two mutations remains unclear. We speculated that L842P, similarly to the reported mutations $\mathrm{P} 726 \mathrm{~L}, \mathrm{~S} 346 \mathrm{I}$ and $\mathrm{A} 286 \mathrm{~V}$ in TMD, may interact with residues in the core of $A B C B 4$, resulting in impaired function (12). Likewise, variation V1051A may cause ATP binding disorder, which is essential for $A B C B 4$-mediated PC extrusion, in a manner similar to mutation S1076C located in NBD (13). We also analyzed the predictive mutation structure and found that the substitution of two amino acids has no impact on the secondary structure of the protein, which may result in a partial residual of protein function. This may be the reason why the patient had a late-onset manifestation.

It is increasingly recognized that genetic variations in $A B C B 4$ cause several cholestatic liver diseases including PFIC3, ICP, LPAC, and DILI $(11,18,19)$. Moreover, genomic studies have shown a link between ABCB4 dysfunction and hepatobiliary malignancies $(20,21)$. PFIC3 represents a severe form of $\mathrm{ABCB} 4$ deficiency. ICP is the most common primary liver disease during pregnancy and characterized by maternal pruritus and increased serum bile acid concentrations (22). LPAC is a peculiar form of intrahepatic cholelithiasis occurring in young adults, which may also recur after cholecystectomy. LPAC and ICP are generally thought to be caused by monoallelic mutations, while PFIC3 is caused by homozygotes or compound heterozygous variant of $A B C B 4$ (11). Furthermore, synonymous, missense, or truncating variants account for ICP and LPAC; nonsense mutations lead to PFIC3 (23-25). Currently, the molecular mechanisms underlying such a wide range of phenotype heterogeneity are not fully understood.

Poupon et al. have reported that an LPAC patient with an $A B C B 4$ homozygous missense mutation (p.Arg47Gln) eventually developed cholangiocarcinoma (26). A large cohort study on LPAC showed that LPAC syndrome is more frequent in women and highly associated with ICP (23). In the current study, two sisters had the same $A B C B 4$ genotype as the patient, but one of them suffered from ICP and the other had asymptomatic gallstones. The above evidence suggests that the same genotype may result in different phenotypes or overlapping disease manifestations. A possible explanation is that a multifactorial etiology including hormonal, environmental, and genetic factors contributes to the development of ABCB4 deficiency-associated diseases. In addition, the patient's parents carried a monoallelic mutation in $A B C B 4$. His mother had gallstones and underwent cholecystectomy, whereas his father was healthy. This suggests that the mutation L842P carried by his mother has a greater impact on ABCB4 function. Even though genotype-phenotype correlations are often weak, the detection of genetic variants might be helpful for the diagnosis and counseling of individual patients with ABCB4 deficiency-associated diseases and their families. Moreover, some patients without detectable variants presented similarly to patients with variants, indicating that yet unexplored regions of the $A B C B 4$ and other genes may be involved (23). Therefore, genetic testing can be useful to elucidate the molecular mechanisms of this group of diseases and discover new potentially pathogenic genes. Further, considering the cost and the willingness of the patient's family, we did not detect other genes involved in biliary diseases other than $A B C B 4$, which may partially explain the difference between siblings. The patient and his family need continued follow-up.

In conclusion, this study demonstrates that a novel compound heterozygous mutation L842P/V1051A in $A B C B 4$ may cause PFIC3 in the pedigree. Our results also reveal inconsistence between genotypes and phenotypes, showing significant diversity. The effect of novel variations on protein function needs further exploration. 


\section{Acknowledgments}

We would like to thank Shuang Liu and Li Bai for their help in polishing the language of our paper.

Funding: This study was supported by Beijing Municipal Science and Technology Project (No. Z171100002217070), National Key R\&D Program of China (No. 2017YFA0103000), National Science and Technology Key Project on "Major Infectious Diseases such as HIV/ AIDS, Viral Hepatitis Prevention and Treatment" (No. 2012ZX10002004-006, No. 2017ZX10203201005, 2017ZX10201201, No. 2017ZX10202203-006001 and No. 2017ZX10302201-004-002), "Beijing Muncipal Administration of Hospitals" Ascent Plan (No. DFL20151601), Beijing Municipal Administration of Hospitals Clinical Medicine Development of Special Funding Support (No. ZYLX201806 and ZYLX202125), the Digestive Medical Coordinated Development Center of Beijing Municipal Administration of Hospitals (No. XXZ0503), Beijing Municipal Natural Science Foundation (7202068), and the You An fund for liver diseases and AIDS (YNKTTS201801189).

\section{Footnote}

Reporting Checklist: The authors have completed the CARE reporting checklist. Available at http://dx.doi.org/10.21037/ atm-20-3747

Peer Review File: Available at http://dx.doi.org/10.21037/ atm-20-3747

Conflicts of Interest: All authors have completed the ICMJE uniform disclosure form (available at: http://dx.doi. org/10.21037/atm-20-3747). The authors have no conflicts of interest to declare.

Ethical Statement: The authors are accountable for all aspects of the work in ensuring that questions related to the accuracy or integrity of any part of the work are appropriately investigated and resolved. All procedures performed in studies involving human participants were in accordance with the ethical standards of the Institute Ethical Committee of Beijing You An Hospital, Capital Medical University, Beijing, China and with the Helsinki Declaration (as revised in 2013). Written informed consent was obtained from the patients for publication of this manuscript and any accompanying images.
Open Access Statement: This is an Open Access article distributed in accordance with the Creative Commons Attribution-NonCommercial-NoDerivs 4.0 International License (CC BY-NC-ND 4.0), which permits the noncommercial replication and distribution of the article with the strict proviso that no changes or edits are made and the original work is properly cited (including links to both the formal publication through the relevant DOI and the license). See: https://creativecommons.org/licenses/by-nc-nd/4.0/.

\section{References}

1. Vitale G, Gitto S, Vukotic R, et al. Familial intrahepatic cholestasis: New and wide perspectives. Dig Liver Dis 2019;51:922-33.

2. Baker A, Kerkar N, Todorova L, et al. Systematic review of progressive familial intrahepatic cholestasis. Clin Res Hepatol Gastroenterol 2019;43:20-36.

3. Chen HL, Wu SH, Hsu SH, et al. Jaundice revisited: recent advances in the diagnosis and treatment of inherited cholestatic liver diseases. J Biomed Sci 2018;25:75.

4. Sambrotta M, Strautnieks S, Papouli E, et al. Mutations in TJP2 cause progressive cholestatic liver disease. Nat Genet 2014;46:326-8.

5. Gomez-Ospina N, Potter CJ, Xiao R, et al. Mutations in the nuclear bile acid receptor FXR cause progressive familial intrahepatic cholestasis. Nat Commun 2016;7:10713.

6. Qiu YL, Gong JY, Feng JY, et al. Defects in myosin VB are associated with a spectrum of previously undiagnosed low gamma-glutamyltransferase cholestasis. Hepatology 2017;65:1655-69.

7. Bull LN, Thompson RJ. Progressive Familial Intrahepatic Cholestasis. Clin Liver Dis 2018;22:657-69.

8. Andress EJ, Nicolaou M, McGeoghan F, et al. ABCB4 missense mutations D243A, K435T, G535D, I490T, R545C, and S978P significantly impair the lipid floppase and likely predispose to secondary pathologies in the human population. Cell Mol Life Sci 2017;74:2513-24.

9. Stättermayer AF, Halilbasic E, Wrba F, et al. Variants in ABCB4 (MDR3) across the spectrum of cholestatic liver diseases in adults. J Hepatol 2020;73:651-63.

10. Prescher M, Kroll T, Schmitt L. ABCB4/MDR3 in health and disease - at the crossroads of biochemistry and medicine. Biol Chem 2019;400:1245-59.

11. Reichert MC, Lammert F. ABCB4 Gene Aberrations in Human Liver Disease: An Evolving Spectrum. Semin Liver Dis 2018;38:299-307. 
12. Suriawinata AA, Thung SN. Liver Pathology: An Atlas and Concise Guide. America: Demos Medical, 2011.

13. Olsen JA, Alam A, Kowal J, et al. Structure of the human lipid exporter ABCB4 in a lipid environment. Nat Struct Mol Biol 2020;27:62-70.

14. Anzivino C, Odoardi MR, Meschiari E, et al. ABCB4 and ABCB11 mutations in intrahepatic cholestasis of pregnancy in an Italian population. Dig Liver Dis 2013;45:226-32.

15. Degiorgio D, Crosignani A, Colombo C, et al. ABCB4 mutations in adult patients with cholestatic liver disease: impact and phenotypic expression. J Gastroenterol 2016;51:271-80.

16. Gordo-Gilart R, Andueza S, Hierro L, et al. Functional analysis of ABCB4 mutations relates clinical outcomes of progressive familial intrahepatic cholestasis type 3 to the degree of MDR3 floppase activity. Gut 2015;64:147-55.

17. Dröge C, Bonus M, Baumann U, et al. Sequencing of FIC1, BSEP and MDR3 in a large cohort of patients with cholestasis revealed a high number of different genetic variants. J Hepatol 2017;67:53-64. </jrn>

18. Jetter A, Kullak-Ublick GA. Drugs and hepatic transporters: A review. Pharmacol Res 2020;154:104234.

19. Goubault P, Brunel T, Rode A, et al. Low-Phospholipid Associated Cholelithiasis (LPAC) syndrome: A synthetic review. J Visc Surg 2019;156:319-28.

Cite this article as: Bai J, Li L, Liu H, Liu S, Bai L, Ning H, Song W, Zou H, Wang X, Chen Y, Zheng S, Duan Z. A novel compound heterozygous mutation in $\mathrm{ABCB} 4$ gene in a pedigree with progressive familial intrahepatic cholestasis 3: a case report. Ann Transl Med 2021;9(5):426. doi: 10.21037/atm20-3747
20. Mhatre S, Wang Z, Nagrani R, et al. Common genetic variation and risk of gallbladder cancer in India: a casecontrol genome-wide association study. Lancet Oncol 2017;18:535-44.

21. Tougeron D, Fotsing G, Barbu V, et al. ABCB4/MDR3 gene mutations and cholangiocarcinomas. J Hepatol 2012;57:467-8.

22. Chappell LC, Bell JL, Smith A, et al. Ursodeoxycholic acid versus placebo in women with intrahepatic cholestasis of pregnancy (PITCHES): a randomised controlled trial. Lancet 2019;394:849-60.

23. Poupon R, Rosmorduc O, Boelle PY, et al. Genotypephenotype relationships in the low-phospholipidassociated cholelithiasis syndrome: a study of 156 consecutive patients. Hepatology 2013;58:1105-10.

24. Dixon PH, Wadsworth CA, Chambers J, et al. A comprehensive analysis of common genetic variation around six candidate loci for intrahepatic cholestasis of pregnancy. Am J Gastroenterol 2014;109:76-84.

25. Gudbjartsson DF, Helgason H, Gudjonsson SA, et al. Large-scale whole-genome sequencing of the Icelandic population. Nat Genet 2015;47:435-44.

26. Khabou B, Trigui A, Boudawara TS, et al. A homozygous ABCB4 mutation causing an LPAC syndrome evolves into cholangiocarcinoma. Clin Chim Acta 2019;495:598-605. 